\title{
The value of serum TPHA titres in selecting patients for lumbar puncture
}

\author{
Y De Silva, M Walzman, M Shahmanesh
}

\begin{abstract}
Between January 1981 and December 1989, 258 patients with positive syphilis serology underwent lumbar puncture. Nine patients had longstanding neurosyphilis and had lumbar punctures performed during the course of their follow-up. Of the remaining 249 only 12 demonstrated the presence of TPHA in the CSF. The serum TPHA titre for eight of these 12 patients, and seven of the nine (two having no titre reported) known neurosyphilis patients at the time of original diagnosis, was 1 in 5120 or more. The four remaining newly found CSF positive patients had serum TPHA titres of 1 in 2560 . We suggest that a serum TPHA titre of 1 in 2560 or greater should be considered as a criterion when selecting patients for lumbar puncture, especially when there are no clinical signs or symptoms.
\end{abstract}

The classical clinical presentations of patients with neurosyphilis is now a rarity in developed countries. ${ }^{1}$ The widespread use of antibiotics and corticosteroids for treating conditions unrelated to syphilis may be responsible for "modified" neurosyphilis as sometimes seen nowadays. ${ }^{12}$ In most of these "modified" cases there appears to be no single or combined clinical or laboratory method which can establish with certainty a diagnosis of active neurosyphilis. ${ }^{3}$

Reports have revealed that screening tests for syphilis on cerebro-spinal fluid (CSF) have been performed on clinical grounds, sometimes without prior blood screening, although, a negative serum Treponema Pallidum Haemagglutination Assay (TPHA) virtually excludes active neurosyphilis. ${ }^{4}$ Lumbar puncture, however, is considered essential in syphilis of uncertain duration, in late symptomatic or late latent stages and in clinical relapse or a fourfold rise in serological titres following treatment. ${ }^{5}$

Although lumbar puncture may be relatively safe,

The Department of Genitourinary Medicine, The General Hospital, Birmingham

Y De Silva, M Walzman, M Shahmanesh it is not without the risk of complications, which can range from being mildly uncomfortable to fatal, ${ }^{6}$ and some physicians nowadays do not recommend it as a routine procedure. ${ }^{78}$

Since a negative test in the CSF for TPHA excludes neurosyphilis, ${ }^{59}$ the aim of this study was to examine the possibility of finding a serum TPHA titre below which a positive CSF-TPHA was unlikely. If a significant cut-off value for serum TPHA titre could be found, this would reduce the need for performing routine lumbar punctures on many asymptomatic patients with positive syphilis serology and no clinical findings.

\section{Patients and methods}

We reviewed retrospectively the notes of 258 patients who underwent lumbar puncture between 1981, when serum TPHA titre levels were first recorded, and December 1989, at the General Hospital, Birmingham. Nine patients had previously diagnosed neurosyphilis and lumbar punctures were performed in the course of their follow-up between the above dates, either as a routine or because of persistently high serum TPHA titres. The remaining patients were those who agreed to lumbar puncture on the basis of clinic policy in regard to offering lumbar puncture to all seropositive patients who were considered to have late (more than two years) syphilis irrespective of whether neurological signs or symptoms were present or not. Apart from two patients who had secondary syphilis, all the others were classified as late latent.

Information recorded included ethnic origin, age, abnormal neurological findings, blood results for the Venereal Diseases Research Laboratory (VDRL) test, the TPHA and the Fluorescent Treponemal Antibody Absorption (FTA-ABS) test, and the corresponding cerebrospinal fluid results for VDRL, TPHA, FTA-ABS, protein and cells when available. The blood and CSF results for the nine known neurosyphilis patients were recorded when taken at the time of the original diagnosis.

The TPHA test was performed in two laboratories. We have reported the values of the laboratory which consistently reported the lower titre levels. This laboratory performed the TPHA 
Table 1 Ethnic distribution and age of study population in relation to serum and CSF TPHA

\begin{tabular}{|c|c|c|c|c|c|c|}
\hline & \multirow[b]{2}{*}{$\begin{array}{l}\text { Ethnic origin } \\
\text { (Numbers) }\end{array}$} & \multirow[b]{2}{*}{$\begin{array}{l}\text { Mean age } \\
(Y r) \text { (Range) }\end{array}$} & \multicolumn{4}{|c|}{ Serum TPHA titres - patient numbers } \\
\hline & & & $\begin{array}{l}1: 5120 \\
\text { or greater }\end{array}$ & $1: 2560$ & $\begin{array}{l}1: 1280 \\
\text { or less }\end{array}$ & $\begin{array}{l}\text { Not } \\
\text { recorded }\end{array}$ \\
\hline \multirow[t]{2}{*}{$\begin{array}{l}\text { CSF-TPHA negative } \\
\text { (Total } 237 \text { patients) }\end{array}$} & $\begin{array}{l}\text { Caucasian } \\
\text { (39) }\end{array}$ & \multirow{2}{*}{$\begin{array}{l}47 \cdot 5 \\
(22-76) \\
54 \cdot 3 \\
(29-74)\end{array}$} & 6 & 5 & 28 & - \\
\hline & $\begin{array}{l}\text { Non-Caucasian } \\
\text { (198) }\end{array}$ & & 20 & 17 & 161 & - \\
\hline \multirow[t]{2}{*}{$\begin{array}{r}\text { CSF-TPHA positive } \\
\text { (Total } 21 \text { patients) }\end{array}$} & $\begin{array}{l}\text { Caucasian } \\
\text { (13) }\end{array}$ & $\begin{array}{l}44 \\
(38-62)\end{array}$ & 8 & 3 & - & 2 \\
\hline & $\begin{array}{l}\text { Non-Caucasian } \\
(8)\end{array}$ & $\begin{array}{l}49 \cdot 5 \\
(41-58)\end{array}$ & 7 & 1 & - & - \\
\hline
\end{tabular}

test according to the instructions of the manufacturer (Fujirebo Inc of Tokyo, Japan), and the screening dilution was 1 in 80 . In more recent years the TPHA titrations were not performed beyond a dilution of 1 in 5120, however, previously it was the laboratory policy to continue the titrations beyond this dilution, thus explaining the one value of 1 in 10240 achieved for one of the nine previously diagnosed neurosyphilis patients. The reported titre is that of the highest dilution showing at least $50 \%$ haemagglutination. The variability of the TPHA test at this laboratory is never more than one doubling dilution above or below the reported value.

\section{Results}

The majority of the patients under study were noncaucasian (table 1). Of the 206 non-caucasians 54 had clinical evidence or past history suggestive of yaws. Of the 52 Caucasian patients, 14 had blood TPHA titres of 1:5120 or greater and of these eight had positive CSF-TPHA.

Of the 21 patients with positive CSF-TPHA, 12 demonstrated some neurological abnormality, while of the nine remaining patients, two previously diagnosed neurosyphilis cases and seven of the 12 newly reported cases, showed no abnormalities. Within the

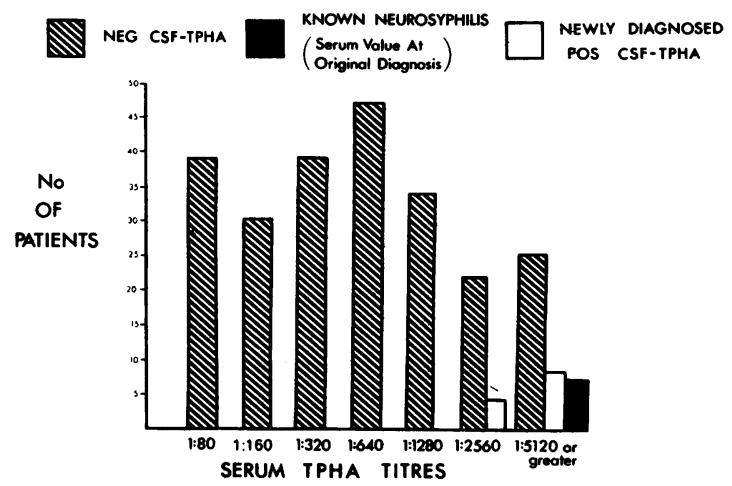

Figure Numbers of patients at each serum TPHA tire and their CSF-TPHA status.

group of 12 patients with abnormalities, one had had a cerebrovascular accident, one suffered from deafness, six had pupillary changes, one had blackouts and dizzy spells, one had extensor-plantar reflexes and there were two with tabes dorsalis.

The figure shows the serum TPHA titres for 258 patients, two of the previously diagnosed neurosyphilis patients not having titres recorded at the time of the original diagnosis and so they are not represen-

Table 2 Laboratory investigations of the nine previously diagnosed neurosyphilis patients at the time of the original diagnosis

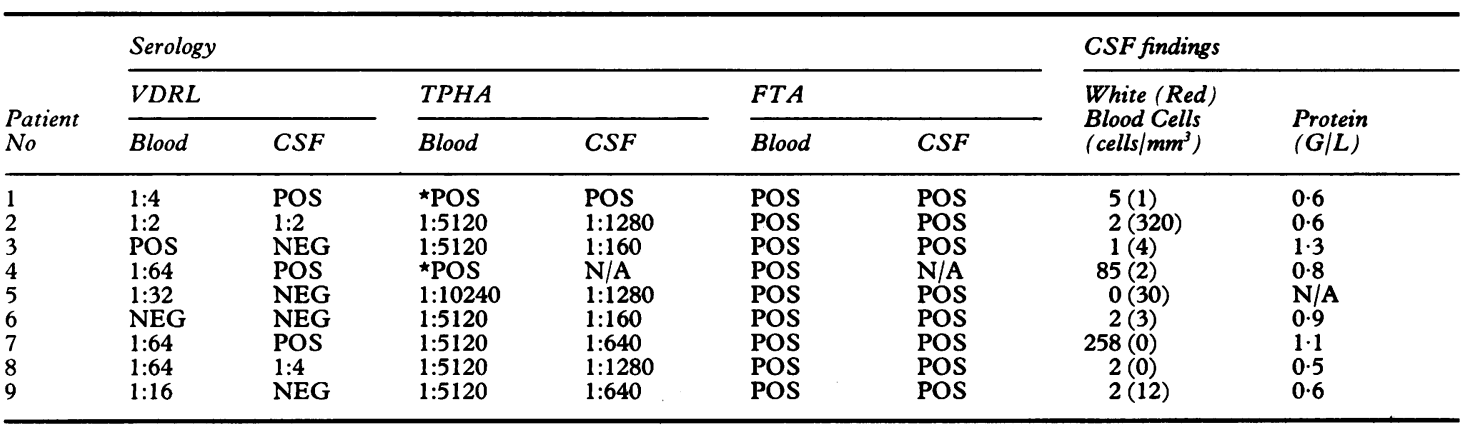

*No titres reported.

N/A, Not available. 
Table 3 Laboratory investigations of newly diagnosed patients with positive CSF-TPHA

\begin{tabular}{|c|c|c|c|c|c|c|c|c|}
\hline \multirow{3}{*}{$\begin{array}{l}\text { Patient } \\
\text { No }\end{array}$} & \multicolumn{6}{|c|}{ Serology } & \multicolumn{2}{|l|}{$C S F$ findings } \\
\hline & \multicolumn{2}{|l|}{$V D R L$} & \multicolumn{2}{|l|}{$T P H A$} & \multicolumn{2}{|l|}{$F T A$} & \multirow{2}{*}{$\begin{array}{l}\text { White (Red) } \\
\text { Blood Cells } \\
\left.\text { (cells } / \mathrm{mm}^{3}\right)\end{array}$} & \multirow{2}{*}{$\begin{array}{l}\text { Protein } \\
(G / L)\end{array}$} \\
\hline & Blood & $C S F$ & Blood & $C S F$ & Blood & $C S F$ & & \\
\hline 1 & $1: 16$ & POS & $1: 5120$ & $1: 320$ & POS & POS & $2(0)$ & 0.6 \\
\hline 2 & POS & NEG & $1: 5120$ & $1: 80$ & POS & POS & $4(0)$ & 0.4 \\
\hline 3 & $1: 4$ & NEG & $1: 5120$ & $1: 80$ & POS & POS & $3(0)$ & 0.5 \\
\hline 4 & NEG & NEG & $1: 5120$ & $1: 80$ & POS & NEG & $2(1)$ & 0.5 \\
\hline 5 & POS & NEG & $1: 2560$ & $1: 160$ & POS & POS & $2(4)$ & 0.4 \\
\hline 6 & $1: 2$ & NEG & $1: 2560$ & $1: 80$ & POS & POS & $2(4)$ & 0.4 \\
\hline 7 & $1: 8$ & NEG & $1: 5120$ & $1: 320$ & POS & POS & 2 (373) & 0.4 \\
\hline 8 & POS & NEG & $1: 2560$ & POS & POS & POS & $2(8)$ & 0.5 \\
\hline 9 & POS & NEG & $1: 2560$ & $1: 160$ & POS & POS & $3(474)$ & 0.8 \\
\hline 10 & NEG & NEG & $1: 5120$ & $1: 640$ & POS & POS & $3(0)$ & 0.4 \\
\hline 11 & $1: 8$ & NEG & $1: 5120$ & $1: 80$ & POS & POS & $4(62)$ & 0.4 \\
\hline 12 & NEG & NEG & $1: 5120$ & $1: 80$ & POS & Doubtful & $3(315)$ & 0.3 \\
\hline
\end{tabular}

ted on the chart. It is noteworthy that both of the former patients had titres of 1:5120 after CSF treponemocidal courses of penicillin on subsequent occasions. From the figure it can be seen that all the patients with positive CSF-TPHA had serum TPHA titres of 1:2560 or greater. There were 47 patients with titres of $1: 2560$ or greater who did not have CSF-TPHA, while the remaining 190 patients without CSF-TPHA had titres less than 1:2560.

Table 2 shows that eight of the nine (the other result not being available) previously diagnosed cases of neurosyphilis, had abnormal CSF protein $(\geqslant 0.5$ $\mathrm{g} / \mathrm{l}$ ) at the time of the original diagnosis and two also had abnormal CSF white blood cell counts. Where the test was available, the patients were also all CSFFTA positive.

In Table 3, the results of the blood and CSF investigations for the 12 newly CSF-TPHA positive patients show that five had abnormal CSF protein and none had abnormal white cell counts. Ten of these were CSF-FTA positive while the other two had either a "doubtful" or negative result.

\section{Discussion}

The classical presentation of neurosyphilis is now rarely seen and in its atypical form the diagnosis may be difficult to establish. The serological tests for syphilis (STS) in blood and any clinical neurological abnormalities all certainly need to be considered. However, examination of the cerebrospinal fluid (CSF) is mandatory before a diagnosis of neurosyphilis can be made. ${ }^{10}$

Lumbar puncture, however, is an invasive procedure not without potential risks to the patient. ${ }^{6}$ Studies assessing the role of lumbar puncture in the evaluation of patients with dementia have concluded that it should not be performed unless there are other indications present. ${ }^{78}$ Hence, we sought a serological titre which could be used as a guide when selecting asymptomatic patients with positive serology for lumbar puncture.

CSF non-treponemal antibody tests are well known to be negative in a proportion of well established cases of neurosyphilis. ${ }^{11}$ The CSF treponemal antibody tests, however, have been found to be consistently positive and CSF-TPHA positivity is essential in order to make the diagnosis. ${ }^{59} \mathrm{We}$ selected the serum TPHA titre as a possible criterion because most laboratories involved in serology have adopted this test, which is considered to be simpler, cheaper, more sensitive and specific than the FTAABS test. ${ }^{12}$

Of 258 lumbar punctures performed in the General Hospital, Birmingham, between January 1981 and December 1989 on patients with positive serological tests for syphilis, only 21 had evidence of abnormal CSF treponemal antibody tests. The remaining 237 therefore could not have had syphilitic involvement of the central nervous system. Of the 21 patients with CSF treponemal antibodies, nine had neurosyphilis diagnosed before 1981 and the most recent lumbar punctures were performed in the course of their follow-up. Hence, of the 249 remaining patients only 12 had a positive CSF-TPHA and therefore could have been considered as potential cases of neurosyphilis.

All nineteen patients in whom serum TPHA titres had been reported at the time of their initial test (two of the previously diagnosed cases of neurosyphilis did not have titres recorded initially but did have subsequent titres of 1:5120) had levels of 1:2560 or greater. If therefore a serum titre of 1:2560 had been taken as a cut-off point for performing a lumbar puncture on the 249 patients (excluding those with known neurosyphilis) in the study only 59 would have been undertaken, thereby reducing the incidence of lumbar puncture by $76 \cdot 3 \%$. Even if a titre of 1:1280 was taken as the cut-off level to allow for a safety margin, the total number of lumbar 
punctures would have been only 81 , still producing a substantial reduction (by $68 \%$ ) in the overall numbers. Apart from eliminating any potential risk to the patients there would have been a substantial financial saving - a study from the USA calculated the cost of performing a lumbar puncture to be in the region of 600 dollars. $^{7}$

We acknowledge that our population in this survey was predominantly non-caucasian and so a percentage possibly had yaws and not syphilis; since there is no distinguishing serological tests to differentiate between these two conditions. However, syphilis is certainly not a rarity in the Caribbean and the Indian sub-continent, and so unless one can be absolutely certain that the diagnosis is of yaws only, the question still arises whether or not a lumbar puncture is indicated in the presence of positive syphilis serology without clinical signs or symptoms. In fact in this study the majority of the newly diagnosed CSFTPHA positive patients were Caucasian.

Although traumatic blood contamination of the CSF can itself convert a TPHA negative CSF into TPHA positive, we have in a separate study (unpublished data) shown that even with a blood TPHA titre of 1:5120, the CSF needs to be macroscopically blood stained before resulting in a false seroconversion of the CSF. However, at higher TPHA titres it is possible that false reactivity in the CSF may result from contamination with quantities of blood that cannot be detected macroscopically. For example, in the case of the CSF-FTA test, seroconverson of $1 \mathrm{ml}$ of CSF requires only $0.8 \mu \mathrm{l}$ blood of $3+$ reactivity and does not cause macroscopic blood staining of the CSF. ${ }^{13}$ None of the 21 patients with a positive CSF-TPHA test in our study had macroscopically visible blood in the CSF and therefore we conclude that the TPHA positivity reported was unlikely to be due to contamination error.

We emphasise that the aim of this study was not the diagnosis of neurosyphilis (especially since we did not have access in these patients to other CSF parameters such as CSF immunoglobulins which are nowadays considered very useful in the diagnosis of neurosyphilis), ${ }^{14}$ but to search for a simple criterion which could justify the performance of a lumbar puncture in asymptomatic patients. We conclude from our study that a serum TPHA titre of $1: 2560$ or above should be considered as a criterion for performing lumbar puncture on the asymptomatic patient with late syphilis (more than two years duration). Finally, since patients with both syphilis and human immunodeficiency virus (HIV) infection presents a far more complex subject, ${ }^{15}$ we feel more study is required in this group before we could advocate our above criterion for performing lumbar punctures.

Address for correspondence: Dr Y De Silva, Department of Genitourinary Medicine, Coventry and Warwickshire Hospital, Stoney Stanton Road, Coventry CV1 4FH.

1 Anonymous. Modified Neurosyphilis. (Editorial). $\mathrm{Br}$ Med J 1978;2:647-8

2 Kolar OJ, Burkhart JE. Neurosyphilis. Br J Venereal Dis 1977;53:221-5.

3 Jordan K. More on Neurosyphilis. West J Med 1982;136:166-7.

4 Anonymous. Routine tests for Syphilis on Cerebrospinal Fluid. (Leading Article). Lancet 1977;ii:595.

5 Robertson DHH, McMillan A, Young H. Diagnosis of Syphilis. In: Robertson DHH, McMillan A, Young H. Eds. Clinical Practice in Sexually Transmissible Diseases. Edinburgh: Churchill Livingstone 1989:118-35.

6 Marton KI, Gean AD. The Spinal Tap: A new look at an old test. Ann Intern Med 1986;104:840-8.

7 Becker PM, Feussner JR, Mulrow CD, Williams BC, Vokaty $\mathrm{KA}$. The role of lumbar puncture in the evaluation of dementia: The Durham Veterans Administration/Duke University Study. J Am Geriatr Soc 1985;33:392-6.

8 Hammerstrom DC, Zimmer B. The role of lumbar puncture in the evaluation of dementia: The University of Pittsburgh Study. J Am Geriatr Soc 1985;33:397-400.

9 Luger A, Schmidt BL, Steyrer K, Schonwald E. Diagnosis of neurosyphilis by examination of the cerebrospinal fluid. $\mathrm{Br} J$ Venereal Dis 1981;57:232-7.

10 Dijkstra JWE. Asymptomatic neurosyphilis. Int J Dermatol 1983;2:581-9.

11 Harner RE, Smith JL, Israel CW. The FTA-ABS test in late syphilis. JAMA 1968;203:545-8.

12 Luger A, Schmidt B, Spendlingwimmer I, Horn F. Recent observations on the serology of syphilis. $\mathrm{Br} J$ Venereal Dis 1980;56:12-6.

13 Davis LE, Sperry S. The CSF-FTA test and the significance of blood contamination. Ann Neurol 1979;6:68-9.

14 Gibowski M, Machonko T. Immunoglobulins in the cerebrospinal fluid of patients with late syphilis. $\mathrm{Br} J$ Venereal Dis $1981 ; 57: 20-4$.

15 Hook EW III. Syphilis and HIV infection. J Infect Dis 1989;160:530-4.

Accepted for publication 8 October 1990 